\title{
Synovial osteochondromatosis of the wrist joint: A case report
}

\author{
HOUYUN GU ${ }^{1 *}$, WEI LI ${ }^{1 *}$, MIN DAI $^{1}$, BIN ZHANG $^{1}$, HUCHENG LIU $^{2}$ and YI DING $^{1}$ \\ ${ }^{1}$ Department of Orthopedics, Artificial Joints Engineering and Technology Research Center of Jiangxi Province; \\ ${ }^{2}$ Multidisciplinary Therapy Center of Musculoskeletal Tumors, \\ The First Affiliated Hospital of Nanchang University, Nanchang, Jiangxi 330006, P.R. China
}

Received December 12, 2014; Accepted December 15, 2015

DOI: $10.3892 / \mathrm{ol} .2016 .4106$

\begin{abstract}
Synovial osteochondromatosis is a rare condition in which multiple cartilaginous nodules proliferate within the synovial membranes of joints, tendon sheaths or bursae. In general, a complete synovectomy is an effective method to treat this disease. Commonly involved joints are the knee, glenohumeral joint, elbow, hip and ankle, although any articulation may be affected. However, synovial osteochondromatosis occurs rarely in the wrist, and there have been a lack of reports of this occurrence in the literature. The current study presents a case of synovial osteochondromatosis in a 33-year-old man, who was admitted in 2014 with the symptom of swelling of the left wrist joint for 2 years. The swelling had become increasingly painful over the previous 2 months. Physical examination revealed local tenderness and a soft pliable mass, with no involvement of the skin and with moderate pain. X-ray, computed tomography and magnetic resonance imaging of the left wrist revealed a lump at the volar radial side of the left wrist joint without any bone erosion. The lesion was subsequently excised. Histological examination resulted in a diagnosis of osteochondromatosis, which was not considered prior to the surgery. The present case was reported with the aim of analyzing the clinical, imaging characteristic and therapeutic modalities of synovial osteochondromatosis of the wrist. While there was no evidence of recurrence for the subsequent 4 months of post-operative follow-up in the present case, the long-term efficacy of surgical excision requires extended observation.
\end{abstract}

\section{Introduction}

Synovial osteochondromatosis is a benign rare disease, which is characterized by synovial metaplasia and hyperplasia, in

Correspondence to: Professor Min Dai, Department of Orthopedics, Artificial Joints Engineering and Technology Research Center of Jiangxi Province, The First Affiliated Hospital of Nanchang University, 17 Yong Wai Zheng Street, Nanchang, Jiangxi 330006, P.R. China

E-mail:250132519@qq.com

*Contributed equally

Key words: synovial osteochondromatosis, wrist joint, surgical excision, cartilaginous nodules which cartilaginous nodule shedding of loose bodies into the synovial cavity is frequently observed (1-4). It's exact incidence is unknown $(5,6)$. The knee joint is the most common articulation involved, followed by the hip, elbow and shoulder. However, the wrist is rarely affected (7-9). One retrospective case series reported a prevalence of $7.5 \%$ in the wrist, compared with $74 \%$ in the knee (10). Our literature review revealed $\sim 29$ cases of synovial osteochondromatosis have been reported in the wrist. The primary clinical manifestations of synovial osteochondromatosis are non-specific, including swelling, pain, a palpable mass, tenderness and restricted movement of the joint, which may develop slowly over several years $(4,11)$. The majority of patients affected by synovial osteochondromatosis are primarily in the third or fifth decade of life (5). Furthermore, the incidence in men is 3-5 times greater than that in women (12). In general, treatment of synovial osteochondromatosis consists of surgical excision of the diseased synovium and cartilaginous nodules, which frequently achieves a satisfactory result $(13,14)$.

Although no comparative studies for the wrist have been performed, removal of loose bodies and synovectomy of the knee leads to positive results for function, pain and control of synovitis in $90 \%$ of subjects (15). However, synovial osteochondromatosis may be locally aggressive with a tendency to recur, but has no metastatic potential. Malignant transformation of preexisting primary synovial osteochondromatosis to synovial chondrosarcoma is recognized to be a rare event with reports estimating the incidence to be in the range of $1-7 \%$ $(6,16)$. Overall, synovial osteochondromatosis is a benign disease, and prognosis following removal of the nodules is reported as excellent.

In order to analyze the clinical, imaging characteristic and therapeutic modalities of synovial osteochondromatosis of the wrist, and to prevent orthopedic surgeons from misdiagnosis that could lead to a delay in treatment, the relevant literature was reviewed and a rare case of synovial chondromatosis in the wrist was reported in the present study. Written informed consent was obtained from the patient for publication of this study.

\section{Case report}

A 33-year-old male patient presented at the First Affiliated Hospital of Nanchang University (Nanchang, China) on July 3, 2014, with the symptom of swelling of the left wrist 

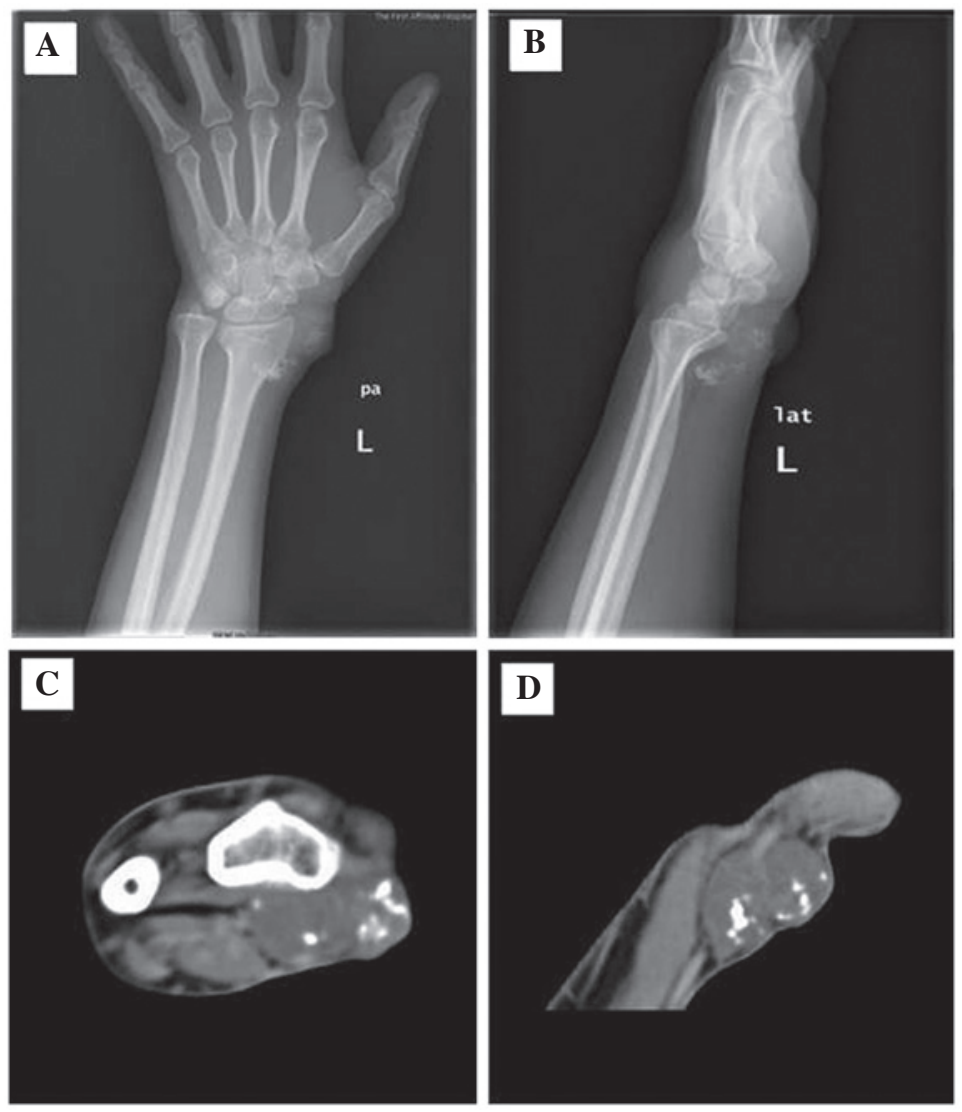

Figure 1. (A and B) Original plain radiograph showing a soft-tissue lump over the volar radial aspect on the inside of the left wrist joint, without any erosion of the adjacent cortex. (C and D) Pre-operative computed tomography scans revealing a lesion within the soft tissue of the radial side of the left wrist joint, measuring $\sim 44 \times 31 \mathrm{~mm}$. The articular surface was smooth, without evidence of any ossification or erosion of the adjacent cortex and without joint effusions. However, the mass presented with low uneven density, accompanied by punctate or ringed calcification, while its boundary remained distinctive.

joint for $\sim 2$ years, which had become increasingly painful over the previous 2 months. There were no systemic symptoms, including weight loss and night sweats, prior to the patient's hospitalization, and the patient had not experienced any previous tumors. The patient was otherwise healthy with a benign medical history, and had no history of previous surgeries. There was no history of trauma to the patient's left wrist joint. Physical examination revealed local tenderness and a soft pliable mass, with no involvement of the skin and little pain; however, there was no profound restriction of wrist joint motion. Therefore, the patient was referred to the Department of Orthopedics (The First Affiliated Hospital of Nanchang University, Nanchang, China) for additional investigation of the lump in the left wrist joint on July 5, 2014. A mass was detected using X-ray imaging (Fig. 1A and B), which revealed a soft-tissue lump over the volar radial side of the left wrist joint, with no erosion of the adjacent cortex. Pre-operative blood test results, including those for tumor markers, were normal. Computed tomography (CT) imaging (SOMATOM Sensation 16; Siemens AG, Munich, Germany) revealed a lesion within the soft tissue of the radial side of the left wrist joint, measuring $\sim 44 \times 31 \mathrm{~mm}$. The articular surface of the lesion was smooth, with no indication of any ossification or erosion of the adjacent cortex. No joint effusion was observed, however, the mass exhibited a low uneven density, accompanied by punctate or ringed calcification bodies, while the boundary of the lesion was distinctive (Fig. 1C and D). Magnetic resonance imaging [MRI; Magnetom Trio, A Tim System, ultra high field MRI (3.0T); Siemens AG] was subsequently performed to investigate the lesion. This revealed a mass expanding into the intra-articular radial side of the joint and around the synovial membrane. The mass demonstrated long signal intensity on T1-weighted images (WI) and a reduced intensity on T2WI. Fat suppression sequences revealed a high signal, which demonstrated a creeping proliferation and a clear boundary. There were no abnormal signals over the adjacent bone, however, a small number of joint effusions were observed (Fig. 2A-C).

As the patient complained of continuously increasing pain, which was caused by the lesion, surgical joint exploration and synovial biopsy were recommended. There were no clear signs of surgical contraindications. Initially, sterile drapes were routinely draped, exposing the surgical field following successful brachial plexus anesthesia. The surgery was performed with an approach that combined a front and rear incision of the left wrist joint. Subcutaneous tissue, superficial fascia and tendons were separated layer by layer.

It was observed that the radial artery and radial vein were wrapped by the lesion, which originated from the wrist. The lesion was stripped away cautiously and was found to be milky-white in color, with the appearance of several cauliflower-like firm cartilaginous nodules, which were located in the front and back of the wrist joint capsule and partly lined by synovium. However, the nodules adhered loosely to the joint capsule and no evidence of erosion over the adjacent bone was 

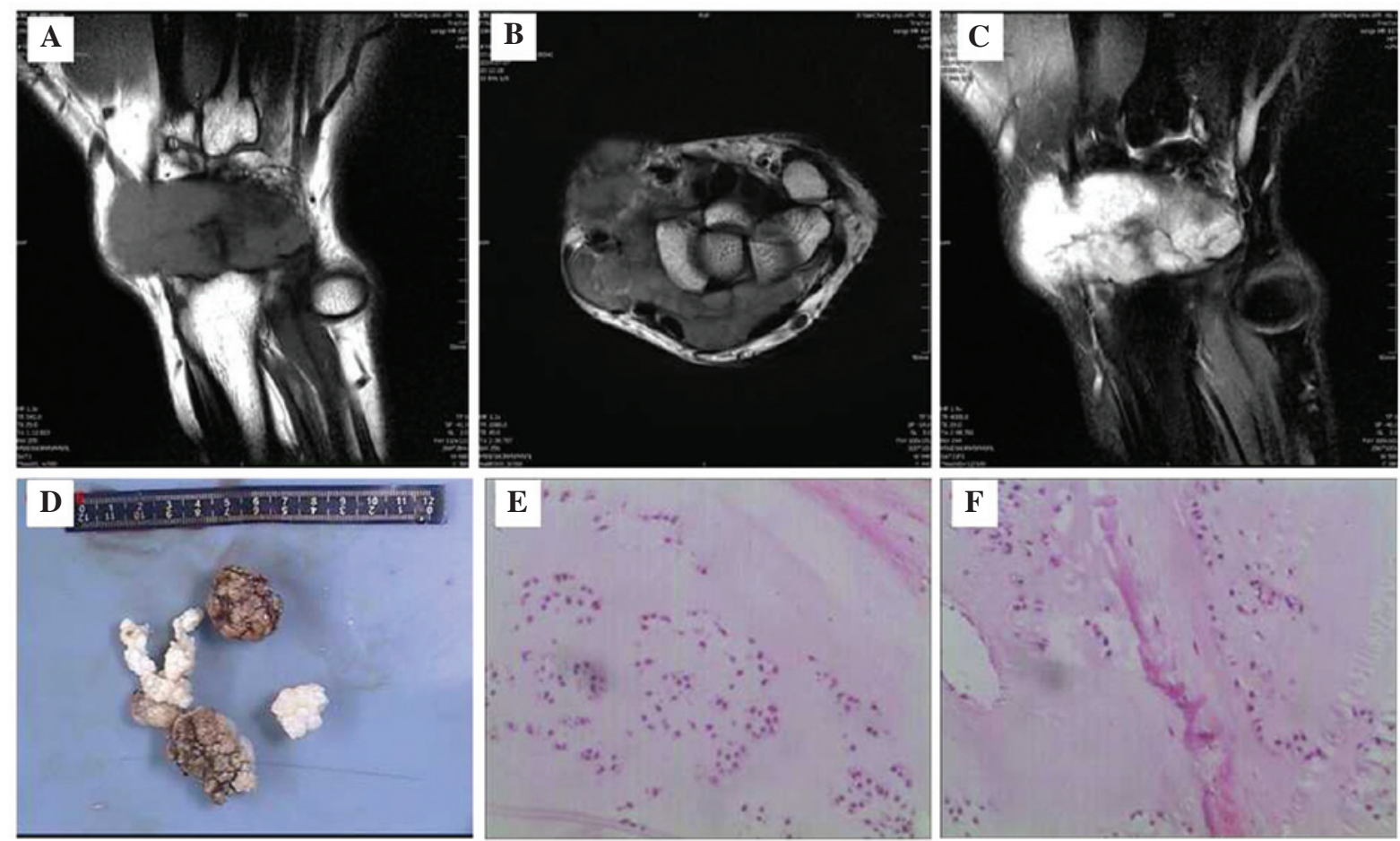

Figure 2. (A-C) Pre-operative magnetic resonance imaging observations revealing a mass expanding into the intra-articular radial side of the wrist and around the synovium. The mass had (A) long signal intensity on T1WI and (B) reduced intensity on T2WI. (C) Fat suppression sequences revealed a high signal, which demonstrated creeping proliferation and a clear boundary. There were no abnormal signals over the adjacent bone, however, a small number of joint effusions were observed. (D) Histological observations. The excised bodies were gray, firm, cartilaginous tissue nodules, which measured $7.5 x 5 x 3 \mathrm{~cm}$. (E and F) Light microscopy revealed small, metaplastic cartilaginous nodules with calcifications. Magnification, x100. Hematoxylin and eosin staining. WI, weighted images.

detected. The mass was excised as completely as possible and a histological examination was performed. The wound was lavaged using a power-pulsed lavage device (Pulsavac ${ }^{\circledR}$ Plus Wound Debridement System Component kit; Zimmer Biomet, Warsaw, IN, USA) and a drainage tube was placed. Finally, each layer of tissue was sutured gradually following sheer hemostasis. During surgery, the patient's estimated blood loss was $100 \mathrm{ml}$ and there was no requirement for a blood transfusion.

Histological examination revealed that the excised bodies were gray, firm, cartilaginous tissue nodules, $\sim 7.5 \times 5 \times 3 \mathrm{~cm}$ in size (Fig. 2D). Tissue sections were prepared as follows: Tissue samples were selected and fixed by immersion in $10 \%$ neutral buffered formalin (Macklin Biochemical Technology Co., Ltd., Shanghai, China) for $8 \mathrm{~h}$. Subsequently, the samples were decalcified (nitrate decalcifying liquid; Gefan Biological Technology Co., Ltd., Shanghai, China) for 12 h, dehydrated using ethanol (Macklin Biochemical Technology Co., Ltd.) and a dewatering machine (PELORIS II; Leica Microsystems $\mathrm{GmbH}$, Wetzlar, Germany) and transparentized (xylene; Macklin Biochemical Technology Co., Ltd.), followed by paraffin embedding (paraffin; Macklin Biochemical Technology Co., Ltd.). Sections ( $3 \mu \mathrm{m}$ thick) were sliced from the paraffin block using a microtome (RM2235; Leica Microsystems $\mathrm{GmbH}$ ), and subsequently deparaffinized (draught drying cabinet; ZBY 149-83; Shanghai Yuejin Medical Instruments Co., Ltd, Shanghai, China) and stained with hematoxylin and eosin (Boster Biological Technology, Pleasanton, CA, USA). Finally, the sections were dehydrated and hyalinized (hyalin; Macklin Biochemical Technology Co., Ltd.), followed by mounting with Permount ${ }^{\mathrm{TM}}$ mounting medium (Boster
Biological Technology). Histopathological assessment under light microscopy (Eclipse Ci-E; Nikon Corp., Tokyo, Japan) revealed small, metaplastic cartilaginous nodules accompanied by calcifications (Fig. 2E and F). Therefore, the tumor was diagnosed as synovial osteochondromatosis.

The associated symptoms improved significantly following excision of the lesion. The patient underwent follow-up for 1.5 years and no residual or recurrent lesions were observed, as well as no evidence of malgnancy.

\section{Discussion}

Synovial osteochondromatosis of the wrist joint is a rare benign tumor, characterized by the presence of multiple cartilaginous nodules within the synovial or tenosynovial membrane $(4,13,17)$. Occasionally, the mass may proliferate beyond the joint and into the adjoining soft tissue. The exact etiology of synovial osteochondromatosis remains to be elucidated. However, Milgram (13) divided it into 3 histological phases based on the maturation of the lesion: i) Active intrasynovial disease with no appearance of loose bodies; ii) loose bodies and active synovial disease; and iii) multiple osteochondral bodies without intrasynovial disease. The present patient was in the second phase of the disease.

A history of trauma does not increase the probability of developing synovial osteochondromatosis in the wrist joint (12). In general, surgical excision of the neoplasm frequently achieves satisfactory results, and post-operative follow-up is required in case of recurrence and potential malignant transformation to chondrosarcoma $(1,18-20)$. Secondary degenerative 
osteoarthritis is the primary complication of synovial osteochondromatosis due to chronic mechanical stimulation and articular cartilage destruction by loose bodies (20). Due to the low incidence and non-specific symptoms of synovial osteochondromatosis, diagnosis of this disease may be difficult, and increased attention should be given to the differential diagnosis of wrist tumors or other osteopathies, including rheumatoid arthritis, secondary synovial osteochondromatosis, chondrocalcinosis, synovial chondrosarcoma and malignant fibrous histiocytoma (18). Notably, the standard method of diagnosis for synovial osteochondromatosis is tissue biopsy followed by pathological examination. In clinical practice, imaging studies are frequently suggested to assist with differentiation between benign and malignant space-occupying lesions $(18,19)$. However, it must be highly emphasized that clinicians should fundamentally depend on clinical examination and imaging during the pre-operative diagnosis of synovial osteochondromatosis.

The most effective treatment method for synovial osteochondromatosis is total synovectomy, with removal of loose cartilaginous nodules, for patients exhibiting constant symptoms $(11,20,21)$. Recurrence following resection has been reported and is most likely due to incomplete excision; therefore, surgeons should ensure a complete synovectomy is performed (22). In addition, although the rate of malignant degeneration and recurrence is low, it is useful to follow up the disease post-operatively.

In general, roentgenographic procedures identify non-specific results, including the presence of a soft-tissue mass, an indefinite quantity of scattered calcifications or a radiopaque tumor. This imaging method relies on calcification or ossification of cartilaginous bodies in the mass (23). Additional radiographic features, including bone erosion, occasional osteoarthritis, novel bone formation and regional osteoporosis have been reported $(17,24)$. Although the metaplastic process of the synovium is self-limiting, multiple loose cartilaginous bodies may lead to mechanical damage of the joint, which may result in early degenerative arthritis (23). CT and MRI are able to provide detailed detection of intra-articular calcifications suggestive of loose bodies.

In conclusion, the current study reported a case of synovial osteochondromatosis originating from the synovium of the wrist joint. The study analyzed the clinical, imaging characteristic and therapeutic modalities of synovial osteochondromatosis, aiming to allow clinicians to improve their performance when encountering patients with lesions of the wrist. As a result of achieving a differential diagnosis of synovial osteochondromatosis, which allows a definite pre-operative diagnosis, a successful synovectomy may be achieved, assisting with improvement of the pain and swelling in symptomatic patients.

\section{Acknowledgements}

The present study was supported by Gan-Po Talents Project 555 of Jiangxi Province and The Spark Program of the Health Department of Jiangxi Province (grant no. 20116007).

\section{References}

1. Wittkop B, Davies AM and Mangham DC: Primary synovial chondromatosis and synovial chondrosarcoma. Eur Radiol 12: 2112-2119, 2002.

2. McLennan MK and Margolis M: Radiology rounds. Synovial osteochondromatosis. Can Fam Physician 40: 1398, 1994.

3. Wong SH, Salama S and Thoma A: Synovial chondromatosis of the hand: Three case reports and literature review. Can J Plast Surg 11: 47-52, 2003.

4. McInnes CW and Goetz TJ: Management of synovial osteochondromatosis of the distal radioulnar joint with imaging features consistent with malignancy. Case Rep Orthop 2013: 589631, 2013.

5. Reverté Vinaixa MM, Singh R, Monyart JM, Llado GD, Dominguez MP, Feliu EC, Nardi Vilardaga $J$ and Palou EC: Wrist synovial chondromatosis: Case report and literature review. Hand Surg 17: 233-238, 2012.

6. Evans S, Boffano M, Chaudhry S, Jeys L and Grimer R: Synovial chondrosarcoma arising in synovial chondromatosis. Sarcoma 2014: 647939, 2014.

7. Roulot E and Le Viet D: Primary synovial osteochondromatosis of the hand and wrist. Report of a series of 21 cases and literature review. Rev Rhum Engl Ed 66:256-266, 1999.

8. Llauger J, Palmer J, Rosón N, Bagué S, Camins A and Cremades R: Nonseptic monoarthritis: Imaging features with clinical and histopathologic correlation. Radiographics 20 (Suppl): S263-S278, 2000.

9. Boles CA and Ward WG Sr: Loose fragments and other debris: Miscellaneous synovial and marrow disorders. Magn Reson Imaging Clin N Am 8: 371-390, 2000.

10. Maurice H, Crone M and Watt I: Synovial chondromatosis. J Bone Joint Surg Br 70: 807-811, 1988.

11. Chillemi C, Marinelli M and de Cupis V: Primary synovial chondromatosis of the shoulder: Clinical, arthroscopic and histopathological aspects. Knee Surg Sport Traumatol Arthrosc 13: 483-488, 2005.

12. Loonen MP and Schuurman AH: Recurrent synovial chondromatosis of the wrist: Case report and literature review. Acta Orthop Belg 71: 230-235, 2005.

13. Milgram JW: Synovial osteochondromatosis: A histopathological study of thirty cases. J Bone Joint Surg Am 59: 792-801, 1977.

14. Sim FH, Dahlin DC and Ivins JC: Extra-articular synovial chondromatosis. J Bone Joint Surg Am 59: 492-495, 1977.

15. Ogilvie-Harris DJ and Weisleder L: Arthroscopic synovectomy of the knee: Is it helpful? Arthroscopy 11: 91-95,1995.

16. Murphey MD, Vidal JA, Fanburg-Smith JC and Gajewski DA: Imaging of synovial chondromatosis with radiologic-pathologic correlation, Radiographics 27: 1465-1468, 2007.

17. Friedman B, Caspi I, Nerubay J, Huszar M, Ganel A and Horoszowski H: Synovial chondromatosis of the hip joint. Orthop Rev 17: 994-998, 1988.

18. McKenzie G, Raby N and Ritchie D: A pictorial review of primary synovial osteochondromatosis. Eur Radiol 18: 2662-2669, 2008.

19. Hallam P, Ashwood N, Cobb J, Fazal A and Heatley W: Malignant transformation in synovial chondromatosis of the knee? Knee 8: 239-242, 2001.

20. McFarland EG and Neira CA: Synovial chondromatosis of the shoulder associated with osteoarthritis: Conservative treatment in two cases and review of the literature. Am J Orthop (Belle Mead NJ) 29: 785-787, 2000.

21. Park JH, Noh HK, Bada LP, Wang JH and Park JW: Arthroscopic treatment for synovial chondromatosis of the subacromial bursa: A case report. Knee Surg Sports Traumatol Arthrosc 15: 1258-1260, 2007.

22. Floyd W III and Troum S: Benign cartilaginous lesions of the upper extremity. Hand Clin 11: 119-132, 1995.

23. Crotty JM, Monu JU and Pope TL Jr: Synovial osteochondromatosis. Radiol Clin North Am 34: 327-342, 1996.

24. Norman A and Steiner GC: Bone erosion in synovial chondromatosis. Radiology 161: 749-752, 1986. 